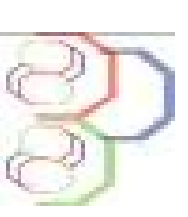

\title{
Caractérisation et évaluation de la phytotoxicité des filtrats de culture de souches de Mycosphaerella fijiensis issues des principales zones de production de bananes en Côte d'Ivoire
}

\author{
AMARI Ler-N'Ogn Dadé Georges Elisée1, PAKORA Gilles Alex², CAMARA Brahima1', LEPENGUE Nicaise \\ Alexis $^{3}$, KONE Daouda ${ }^{1}$ \\ 1 Laboratoire de Physiologie Végétale, UFR Biosciences, Université Félix Houphouët-Boigny d'Abidjan (UFHB), 22 \\ BP 582 Abidjan 22, Côte d'Ivoire ; \\ 2 Laboratoire de Pharmacodynamie Biochimique, UFR Biosciences, Université Félix Houphouët-Boigny d'Abidjan \\ (UFHB), 22 BP 582 Abidjan 22, Côte d'Ivoire ; \\ 3 Laboratoire de Physiologie Végétale et Amélioration des plantes, Unité de recherche Agrobiologie, Université des \\ Sciences et Techniques de Masuku (USTM) ; BP 067 Franceville, Gabon ; \\ *Auteur en correspondance : amariler@yahoo.fr Tel : +225 48075871 / +225 06080463 \\ Original submitted in on $14^{\text {th }}$ August 2018. Published online at www.m.elewa.org on $30^{\text {th }}$ September 2018 \\ http://dx.doi.org/10.4314/jab.v129i1.5
}

\section{RESUME :}

Objectifs : Cette étude a été conduite afin de rechercher la production de toxines par des souches ivoiriennes de M. fijiensis, et d'étudier leur implication dans le développement de la maladie des raies noires (MRN) du bananier, causée par ce champignon. Elle a consisté à déterminer la diversité des métabolites produits par ces isolats fongiques et à évaluer la phytotoxicité de leurs filtrats de culture sur les feuilles de variétés de bananiers présentant au champ un comportement variable à la MRN.

Méthodologie et résultats : Pour réaliser cette étude, une caractérisation physico-chimique des filtrats de culture des souches de $M$. fijiensis a été effectuée. Elle a mis en évidence trois principaux groupes de métabolites secondaires : les saponines, les stérols et terpènes et les alcaloïdes. Le test d'induction de nécroses a été réalisé pour déterminer l'activité phytotoxique des filtrats après leur infiltration dans le limbe des feuilles des bananiers. Les résultats ont montré qu'au bout de 48 heures, seulement certains filtrats de cultures du champignon ont pu induire des nécroses dans les feuilles des variétés de bananiers. La variété partiellement résistante à la MRN (FHIA 23) s'est révélée la plus résistante à la toxicité des filtrats de culture à la différence des variétés très résistante (Calcultta 4) et sensible (Grande Naine) à la maladie.

Conclusion et application des résultats : Les souches de Mycosphaerella fijiensis issues de la Côte d'Ivoire sont capables de produire des métabolites toxiques pour les bananiers. Ces toxines participent au développement des nécroses foliaires chez le bananier et sont tout au moins impliquées dans l'extension de ces symptômes et par conséquent peuvent constituer un outil simple d'évaluation rapide de la résistance des variétés de bananier à la MRN. Un probable contournement de la résistance totale de Calcutta 4 peut être envisagé chez cette variété de bananier par $M$. fijiensis.

Mots clés : métabolites secondaires, Mycosphaerella fijiensis, bananier, phytotoxicité, maladie des raies noires, Côte d'Ivoire. 
Characterization and assessment of the phytotoxicity of culture filtrates of Mycosphaerella fijiensis strains from the main areas of banana production in Côte d'Ivoire

ABSTRACT :

Objective: This study was conducted to investigate the production of toxins by Ivorian strains of $M$. fijiensis, and to study their involvement in the development of banana black leaf streak disease (BLSD) caused by this fungus. It consisted in determining the diversity of the metabolites produced by these fungal isolates and in evaluating the phytotoxity of their culture filtrates on the leaves of the banana varieties presenting in the field a variable behavior to the BLSD.

Methodology and results : To carry out this study, a physico-chemical characterization of the culture filtrates of M. fijiensis strains was carried out. This analysis revealed three main groups of secondary metabolites: saponins, sterols and terpenes and alkaloids. The necrosis induction test was carried out to determine the phytotoxic activity of the filtrates after their infiltration into the banana leaf limb. The results showed that after 48 hours, only certain filtrates from the fungus cultures could induce necrosis in the leaves of banana varieties. The variety partially resistant to the BLSD (FHIA 23) proved to be the most resistant to the toxicity of the culture filtrates unlike the very resistant (Calcultta 4 ) and susceptible (Grande Naine) varieties to the disease.

Conclusion and application of results: Mycosphaerella fijiensis strains from Côte d'lvoire are capable of producing toxic metabolites for banana. These toxins contribute to the development of foliar necrosis in banana and are at least involved in the extension of these symptoms and can therefore be a simple tool for rapid assessment of the resistance of banana varieties to BLSD. A probable circumvention of the total resistance of Calcutta 4 can be considered in this banana variety by $M$. fijiensis.

Keywords: secondary metabolites, Mycosphaerella fijiensis, banana, phytotoxicity, black leaf streak disease, Côte d'Ivoire.

\section{INTRODUCTION}

La maladie des raies noires (MRN) du bananier, provoquée par le champignon Mycosphaerella fijiensis est une maladie qui entraine un desséchement des feuilles et ainsi une perte de rendement pouvant être totale (Carlier et al., 2000 ; Ganry, 2010). La lutte chimique, très importante dans certaines régions du monde, fait aujourd'hui face à une efficacité limitée et engendre des effets néfastes sur l'environnement et la santé humaine, ainsi que l'apparition des souches résistantes aux fongicides (Cañas-Gutiérrez et al., 2009 ; Essis et al., 2010). En outre, l'accessibilité difficile de cette méthode de lutte aux petits producteurs de bananes, dans le contexte actuel de la réduction des pesticides de synthèse, impose la recherche de stratégies de lutte plus écologiques. Parmi les nouvelles stratégies de gestion efficace de la MRN se trouve l'utilisation de variétés résistantes issues de programmes d'amélioration génétique (Vishnevetsky et al., 2011) et la résistance induite par les éliciteurs de défense des plantes (Soler et al., 2102; Amari et al., 2014). Avec ces méthodes, l'utilisation des métabolites secondaires de $M$. fijiensis pourrait constituer un atout majeur pour l'établissement précoce du statut du matériel végétal vis-à-vis de la MRN avant son déploiement en milieu réel. En effet, les métabolites toxiques produits par les microorganismes dont les champignons, sont connus avoir des applications potentielles intéressantes (Harelimana et al., 1997). Ils peuvent être utilisés comme sonde pour étudier la base moléculaire des interactions hôte-parasite et comme moyen pour une sélection rapide et précoce de la résistance, en particulier chez le couple $M$. fijiensisbananier (Amari et al., 2011 et Amari et al., 2014). Les résultats de certains travaux suggèrent de par leur production, leur bioactivité et leur sélectivité visà-vis de la plante hôte, l'hypothèse d'un rôle possible des toxines dans l'infection de M. fijiensis (El Hadrami et al., 2005 ; Amari et al., 2008). Ces composés toxiques contribueraient à l'extension des nécroses et constitueraient tout au moins des déterminants secondaires de l'infection. Cependant, peu de travaux ont été consacrés à l'étude des toxines impliquées dans la pathogenèse de $M$. fijiensis. A ce jour en Côte d'Ivoire où cette maladie 
est très dévastatrice sur les bananiers et bananiers plantains, aucune donnée n'existe sur la production et l'activité des métabolites secondaires des souches de Mycosphaerella fijiensis présentes. Ce travail vise à rechercher chez des isolats de $M$. fijiensis provenant de Côte d'Ivoire la production de métabolites toxiques en vue d'étudier leur implication dans le développement de la maladie des raies noires du bananier. II s'agit de déterminer la diversité des toxines produites par ces isolats fongiques et d'évaluer l'activité phytotoxique des filtrats de culture sur les feuilles de variétés de bananiers présentant une réaction différentielle à $M$. fijiensis.

\section{MATERIEL ET METHODES}

Génotypes de bananiers : Des vivoplants appartenant à trois génotypes de bananiers présentant un comportement variable vis-à-vis de la MRN ont été utilisés au stade moyen de 4 à 5 feuilles dans ce travail. Ce sont: le cultivar très sensible, Grande Naine;

l'hybride tétraploïde partiellement résistant, FHIA 23 ; et le géniteur sauvage très résistant, Calcutta 4 (Traoré, 2008). Ces vivoplants de bananiers ont été obtenus auprès du Centre National de Recherche Agronomique (CNRA).

Tableau 1 : Origine des différentes souches de Mycosphaerella fijiensis utilisées pour la production de toxines

\begin{tabular}{|c|c|c|c|c|c|}
\hline $\begin{array}{l}\text { Code des } \\
\text { isolats }\end{array}$ & $\begin{array}{l}\text { Régions de la } \\
\text { Côte d'Ivoire }\end{array}$ & Localités & $\begin{array}{c}\text { Hôtes } \\
\text { d'isolement }\end{array}$ & $\begin{array}{l}\text { Types de } \\
\text { spore }\end{array}$ & $\begin{array}{c}\text { Dates } \\
\text { d'isolement }\end{array}$ \\
\hline$\delta 124$ & Sud Est & Koukourandoumi & Figue Sucrée & Conidie & Octobre 2008 \\
\hline$\delta 125$ & Sud Est & Koukourandoumi & Figue Sucrée & Conidie & Octobre 2008 \\
\hline$\delta 15$ & Sud & Université Nangui Abrogoua & Plantain & Conidie & Décembre 2008 \\
\hline$\delta 85$ & Sud & Azaguié & Plantain & Conidie & Décembre 2008 \\
\hline$\delta 91$ & Sud & Azaguié & Plantain & Conidie & Décembre 2008 \\
\hline$\delta 98$ & Sud & Abbé & Hybride & Conidie & Décembre 2008 \\
\hline$\delta 100$ & Sud & Abbé & Hybride & Conidie & Décembre 2008 \\
\hline$\delta 102$ & Sud & Abbé & Hybride & Conidie & Décembre 2008 \\
\hline$\delta 104$ & Sud & Abbé & Hybride & Conidie & Décembre 2008 \\
\hline$\delta 106$ & Sud & Adzopé & Plantain & Conidie & Décembre 2008 \\
\hline$\delta 108$ & Sud & Adzopé & Plantain & Conidie & Décembre 2008 \\
\hline$\delta 110$ & Est & Arrah & Plantain & Conidie & Décembre 2008 \\
\hline$\delta 115$ & Est & Arrah & Plantain & Conidie & Décembre 2008 \\
\hline$\delta 35$ & Sud Ouest & Sassandra Corridor & Plantain & Conidie & Novembre 2008 \\
\hline$\delta 123$ & Sud Ouest & Monogaga Carrefour & Plantain & Conidie & Novembre 2008 \\
\hline$\delta 117$ & Sud Ouest & Gnity caillou & Plantain & Conidie & Novembre 2008 \\
\hline$\delta 147$ & Sud Ouest & Grand Zattry & Plantain & Conidie & Novembre 2008 \\
\hline$\delta 23$ & Centre Ouest & Zokougbeu & Dessert & Ascospore & Novembre 2008 \\
\hline$\delta 126$ & Centre Ouest & Tipadipa & Plantain & Conidie & Novembre 2008 \\
\hline$\delta 127$ & Centre Ouest & Tipadipa & Plantain & Conidie & Novembre 2008 \\
\hline$\delta 56$ & Centre Ouest & Daloa & Plantain & Ascospore & Novembre 2008 \\
\hline$\delta 63$ & Ouest & Duekoué & Plantain & Ascospore & Novembre 2008 \\
\hline$\delta 66$ & Ouest & Duekoué & Plantain & Ascospore & Août 2008 \\
\hline$\delta 77$ & Ouest & Man & Plantain & Conidie & Novembre 2008 \\
\hline$\delta 51$ & Ouest & Danané & Plantain & Ascospore & Août 2008 \\
\hline$\delta 52$ & Ouest & Danané & Plantain & Ascospore & Novembre 2008 \\
\hline
\end{tabular}

Souches fongiques: Des souches de M. fijiensis identifiées à partir de la technique classique basée sur la morphologie, ont été utilisées pour la production des phytotoxines. Au nombre de 26, les souches fongiques réparties dans les principales zones de production de bananes en Côte d'lvoire, ont été fournies par la mycothèque du Laboratoire de Physiologie Végétale de I'Université Félix Houphouet-Boigny d'Abidjan (Tableau 
1). Ces isolats issus de culture monospore, ont été à l'origine obtenus à partir de feuilles de bananiers dessert et de bananiers plantain infectées par la MRN.

Préparation des filtrats de culture de $M$. fijiensis: Chaque souche de $M$. fijiensis ont été mise en croissance dans des boîtes de Pétri sur milieu de culture PDA (potato dextrose agar) et les boîtes ont été placées pendant 30 jours à la température ambiante sous une photopériode de 12 heures. Au terme de cette période, une suspension spores/mycélium a été préparée après avoir prélevé et broyé des fragments mycéliens dans $5 \mathrm{ml}$ d'eau distillée stérile. Le milieu de culture V8 a été inoculé avec $3 \mathrm{ml}$ de la suspension aqueuse de chaque souche de $M$. fijiensis et incubé à $25^{\circ} \mathrm{C}$ pendant 14 jours, sous une photopériode de 12 heures. Le milieu V8 a servi à la production abondante de spores (Ganry et al., 2008). Cinq millilitres d'eau distillée stérile ont ensuite été ajoutés à chaque milieu de culture V8 et une suspension de spores a été préparée en grattant doucement la surface des boîtes avec une pipette pasteur incurvée. Un millilitre de la suspension de spores a été utilisé pour inoculer des fioles contenant $125 \mathrm{ml}$ de milieu de culture liquide M1D selon la méthode modifiée de Strobel et al. (1993). Le milieu synthétique M1D a été supplémenté avec de l'eau de coco (1000:12; v/v) étant donné ses effets stimulateurs de la sporulation et la production de phytotoxines (Puch-Ceh et al., 2005). Les fioles ont été ensuite incubées à $25^{\circ} \mathrm{C}$ sous une photopériode de 12 heures et soumises à une agitation continue à $120 \mathrm{rpm}$ pendant 28 jours. Le bouillon de culture a été séparé $\mathrm{du}$ mycélium par filtration à travers 4 couches de percale stérile (étamine) à la fin de la période de culture. L'extrait obtenu pour chaque souche de $M$. fijiensis a constitué le filtrat de culture. Des préparations sans inoculation de spores fongiques ont subi le même traitement et ont servi de témoin (To). Trois répétitions ont été effectuées et ont permis de réaliser 3 préparations pour chacune des 26 souches de M. fijiensis testées.

Caractérisation physico-chimique des filtrats de culture de M. fijiensis : Après l'obtention du bouillon de culture, les mesures des paramètres physico-chimiques ont porté sur la masse du mycélium, le rendement en extrait organique brut, le $\mathrm{pH}$ et la recherche de métabolites secondaires du filtrat de culture sur chaque préparation de filtrat réalisée. La masse du mycélium a été déterminée après séchage à l'étuve à $50^{\circ} \mathrm{C}$ pendant $24 \mathrm{~h}$. Le rendement ( $\mathrm{mg} / \mathrm{l})$ en extrait organique brut a été obtenu à partir de la masse du résidu d'une fraction aliquote de $75 \mathrm{ml}$ du filtrat de culture lyophilisée. Le pH a été mesuré à l'aide d'un pH-mètre (HI 1290 PICCOLO amplified Electrode by HANNA) à $25^{\circ} \mathrm{C}$. Les mesures ont porté sur les trois préparations de filtrats réalisées pour chacune des souches de M. fijiensis mises en culture. Pour la mise en évidence des différents groupes de métabolites secondaires, afin d'éviter la trop grande variabilité de réaction entre les filtrats issus d'une même souche, les trois préparations ont été combinées en un filtrat unique. Un screening chimique a été effectué sur les filtrats de culture grâce aux réactions caractéristiques des différents composés recherchés selon le protocole décrit par Tarik et al., (2011). Les métabolites secondaires recherchés étaient: les saponosides, les stérols et terpènes, les polyphénols, les quinones, les flavonoïdes, les tanins et les alcaloïdes.

Évaluation de la phytotoxicité des filtrats : La toxicité des filtrats de culture des souches de $M$. fijiensis a été évaluée à partir du test biologique révélant l'induction de nécroses foliaires chez le bananier.

- Infiltration des filtrats de culture dans le limbe foliaire : Des vivoplants de bananiers des variétés Grande Naine, FHIA 23 et Calculta 4 ont été préalablement acclimatés en atmosphère humide et en conditions d'éclairement naturel au sein d'un abri recouvert de film plastique transparent. L'humidité à l'intérieur de l'enceinte a été maintenue à $90 \%$ pendant $48 \mathrm{~h}$ à $25^{\circ} \mathrm{C}$ grâce à une pulvérisation d'eau, afin de favoriser une ouverture optimale des stomates des bananiers. Sur la face inférieure de la deuxième feuille totalement déroulée en dessous du cigare, 20 $\mu l$ de filtrat de culture des souches de M. fijiensis, de filtrat vierge ou d'eau distillée stérile ont été infiltrés. L'opération d'infiltration des différentes solutions a été effectuée à l'aide d'une seringue de $1 \mathrm{ml}$ dont l'aiguille enfoncée dans une gomme percée affleure au niveau de l'épiderme foliaire. Au niveau de chaque feuille traitée quatre sites d'application par solution à infiltrer ont été réalisés à raison de deux par demi-limbe. Les bananiers traités ont été replacés sous l'abri dans les conditions ambiantes. L'ensemble du test a été répété 3 fois.

- Détermination des indices de nécroses : La toxicité des filtrats de culture des souches de $M$ fijiensis a été évaluée $48 \mathrm{~h}$ après l'infiltration des feuilles des bananiers. La sévérité des nécroses formées a été ainsi déterminée à partir d'une échelle visuelle adaptée de celle de Stierle et al., (1991) suivant la surface nécrosée $(\mathrm{Sn})$ par rapport à celle infiltrée $(\mathrm{Si})$. Sur cette échelle les indices de nécroses étaient compris entre 0 et 4 avec : $0=$ absence de nécrose ; $1=\mathrm{Sn}$ inférieure au quart de $\mathrm{Si} ; 2=\mathrm{Sn}$ comprise entre le quart et la moitié de $\mathrm{Si} ; 3=\mathrm{Sn}$ comprise entre la moitié et les trois quarts de $\mathrm{Si} ; 4=\mathrm{Sn}$ 
supérieure aux trois quarts de $\mathrm{Si}$ (Amari et al., 2008). L'indice moyen de nécrose a été déterminé pour l'ensemble des sites infiltrés par filtrat de culture pour chaque souche de M. fijiensis et pour chacune des variétés de bananier testées.

Analyses statistiques : Les données ont été soumises à une analyse de variance à l'aide du logiciel STATISTICA

\section{RESULTATS}

Caractéristiques physiques et chimiques des filtrats de culture des souches de $M$. fijiensis issus de Côte d'Ivoire : Le poids sec de mycélium, le pH et la quantité d'extrait organique brut des filtrats de culture, ont été statistiquement différents entre les souches de $M$. fijiensis étudiées $(P<0,05)$. Les poids secs de mycélium les plus faibles ont été observés avec la souche $\delta 147(0,04 \mathrm{~g})$ qui n'a montré aucune croissance mycélienne visible. Le mycélium produit par la souche $\delta 115$ a été le plus abondant avec une masse de $0,98 \mathrm{~g}$ (Tableau 2). Tous les filtrats ont enregistré un $\mathrm{pH}$ acide. Chez $88,46 \%$ des souches, les $\mathrm{pH}$ des filtrats de culture ont été inférieurs à 5 tandis que seulement $11,53 \%$ de souches ont produit des filtrats ayant des pH supérieurs à 5 (Tableau 2). Comparés au filtrat du milieu non inoculé $(\mathrm{pH}=5,67)$, un $\mathrm{pH}$ en général plus faible a été observée dans les filtrats obtenus avec les milieux de culture inoculés par les souches M fijiensis testées (Tableau 2). Les pH acides de ces milieux inoculés avec le champignon étaient compris entre 1,99 (pour la souche $\delta 108$ ) et 5,27 (pour la souche $\delta 15)$. Les souches $\delta 108$ et $\delta 104$ ont produit des filtrats de culture avec de faibles $\mathrm{pH}$ respectivement 1,99 et 2,84, mais elles ont montré une croissance mycélienne abondante en particulier avec la souche $\delta 108$ et un faible rendement en extrait organique (Tableau 2). A l'inverse,
7.0 afin d'étudier les effets simples et combinés des facteurs souche de $M$ fijiensis et variété de bananiers sur la phytotoxicité des filtrats de culture du champignon. En cas de différence significative, les moyennes ont été comparées selon le test de Newman-Keuls au seuil de 5 $\%$.

les souches $\delta 15, \delta 117, \delta 123$ et $\delta 147$ qui ont produit des filtrats avec des $\mathrm{pH}$ supérieurs à 4,5 et les plus faibles croissances mycéliennes, ont un rendement en extrait organique systématiquement plus élevé (Tableau 2). Les différentes relations pouvant exister entre les caractéristiques physiques ont révélé que seul le poids sec de mycélium est significativement corrélé au $\mathrm{pH}$ ( $\mathrm{r}=$ 0,62 ). Cette corrélation négative indique que ces deux paramètres évoluent en sens inverse et donc à une baisse $\mathrm{du} \mathrm{pH}$ correspond une croissance mycélienne importante et vice versa. Le screening chimique a permis la mise en évidence sur l'ensemble des filtrats de culture, de trois groupes de métabolites secondaires: les saponines, les stérols et terpènes et les alcaloïdes (Tableau 3). Contrairement aux alcaloïdes, les saponosides et le groupe des stérols et terpènes n'ont pas été retrouvés dans tous les filtrats de culture, notamment dans ceux obtenus à partir des souches $\delta 23$, $\delta 56, \delta 123$ (absence de saponosides) et $\delta 56, \delta 115$ (absence de stérols et terpènes). Les tests caractéristiques de ces composés chimiques ont révélé une variabilité de l'intensité de réaction (coloration) des différents filtrats allant des faibles aux fortes intensités de réaction (Tableau 3). 
Tableau 2 : Caractéristiques physiques des filtrats de culture des différentes souches de M. fijiensis en milieu liquide M1D modifié

\begin{tabular}{c|c|c|c}
\hline \multirow{2}{*}{ Souches } & \multicolumn{3}{|c}{ Caractères physiques du filtrat de culture } \\
\cline { 2 - 3 } & $\begin{array}{c}\text { Quantités de } \\
\text { mycélium (g) }\end{array}$ & $\mathbf{p H}$ & $\begin{array}{c}\text { Quantités d'extrait organique brut } \\
\text { (mg/l) }\end{array}$ \\
\hline $\mathbf{T}_{0}$ & $0 \mathrm{~h}$ & $5,67 \mathrm{a}$ & $0 \mathrm{k}$ \\
$\delta 147$ & $0,04 \mathrm{gh}$ & $4,53 \mathrm{ac}$ & $176,13 \mathrm{a}$ \\
$\delta 123$ & $0,08 \mathrm{gh}$ & $4,97 \mathrm{ac}$ & $171,59 \mathrm{a}$ \\
$\delta 117$ & $0,09 \mathrm{gh}$ & $4,98 \mathrm{ac}$ & $142,36 \mathrm{ad}$ \\
$\delta 15$ & $0,11 \mathrm{gh}$ & $5,27 \mathrm{a}$ & $175,79 \mathrm{a}$ \\
$\delta 98$ & $0,12 \mathrm{fh}$ & $4,61 \mathrm{ac}$ & $150,53 \mathrm{ad}$ \\
$\delta 91$ & $0,13 \mathrm{fh}$ & $3,91 \mathrm{ad}$ & $24,24 \mathrm{jk}$ \\
$\delta 110$ & $0,13 \mathrm{fh}$ & $4,74 \mathrm{ac}$ & $112,90 \mathrm{dg}$ \\
$\delta 77$ & $0,16 \mathrm{fh}$ & $4,12 \mathrm{ad}$ & $151,51 \mathrm{ad}$ \\
$\delta 51$ & $0,29 \mathrm{eh}$ & $3,88 \mathrm{ad}$ & $141,72 \mathrm{ad}$ \\
$\delta 63$ & $0,29 \mathrm{eh}$ & $3,30 \mathrm{ad}$ & $121,91 \mathrm{cf}$ \\
$\delta 124$ & $0,35 \mathrm{eh}$ & $4,58 \mathrm{ac}$ & $156,29 \mathrm{ac}$ \\
$\delta 56$ & $0,37 \mathrm{eh}$ & $5,23 \mathrm{ad}$ & $127,33 \mathrm{be}$ \\
$\delta 35$ & $0,41 \mathrm{dg}$ & $4,95 \mathrm{ac}$ & $112,51 \mathrm{dg}$ \\
$\delta 23$ & $0,50 \mathrm{cf}$ & $3,87 \mathrm{ad}$ & $82,83 \mathrm{gh}$ \\
$\delta 126$ & $0,57 \mathrm{be}$ & $3,90 \mathrm{ad}$ & $99,20 \mathrm{eh}$ \\
$\delta 100$ & $0,57 \mathrm{be}$ & $4,37 \mathrm{ac}$ & $153,50 \mathrm{ad}$ \\
$\delta 127$ & $0,63 \mathrm{ae}$ & $3,93 \mathrm{ad}$ & $86,28 \mathrm{fh}$ \\
$\delta 106$ & $0,64 \mathrm{ae}$ & $3,52 \mathrm{ad}$ & $72,84 \mathrm{hi}$ \\
$\delta 85$ & $0,66 \mathrm{ae}$ & $5,15 \mathrm{ac}$ & $142,81 \mathrm{ad}$ \\
$\delta 52$ & $0,67 \mathrm{ae}$ & $3,64 \mathrm{ad}$ & $166,66 \mathrm{ab}$ \\
$\delta 66$ & $0,76 \mathrm{ad}$ & $4,68 \mathrm{ac}$ & $125,40 \mathrm{be}$ \\
$\delta 125$ & $0,85 \mathrm{ad}$ & $3,21 \mathrm{bd}$ & $156,68 \mathrm{ac}$ \\
$\delta 108$ & $0,91 \mathrm{ab}$ & $1,99 \mathrm{~d}$ & $29,78 \mathrm{jk}$ \\
$\delta 102$ & $0,94 \mathrm{ab}$ & $3,77 \mathrm{ad}$ & $50,36 \mathrm{ij}$ \\
$\delta 104$ & $0,94 \mathrm{ab}$ & $2,84 \mathrm{~cd}$ & $139,95 \mathrm{ad}$ \\
$\delta 115$ & $0,98 \mathrm{a}$ & $3,94 \mathrm{ad}$ & $114,84 \mathrm{cg}$ \\
\hline
\end{tabular}

$\mathrm{T}_{0}=$ milieu non inoculé par $M$. fijiensis. Dans une même colonne, les valeurs suivies d'une même lettre ne sont pas significativement différentes au seuil $\alpha=0,05$ selon le test de Newman-Keuls.

Activités biologiques des filtrats de culture de $M$. fijiensis: Les propriétés biologiques des filtrats de culture des souches de $M$. fijiensis ont été évaluées sur des génotypes de bananiers de sensibilité différente par le test d'induction de nécroses.

Effet des filtrats de culture des souches de $M$. fijiensis sur des génotypes de bananiers présentant une sensibilité différente à la maladie des raies noires : $L$ 'infiltration des filtrats de culture dans les limbes foliaires des différentes variétés de bananiers a révélé une variabilité de réponses. Les nécroses induites sous forme de taches brunâtres ou noirâtres sont caractéristiques des symptômes causés par $M$. fijiensis sur les feuilles de bananiers. Des effets statistiquement différents portant sur la toxicité des filtrats produits par les souches de $M$. fijiensis et sur la sensibilité des génotypes de bananiers, ont été notés. Après 48 heures d'incubation, les filtrats appartenant à 16 souches (soit $61,53 \%$ du total) ont pu induire des nécroses sur les feuilles chez au moins une des variétés de bananiers testées (Figure 1). Les 16 souches de M. fijiensis dont les filtrats de culture ont présenté une toxicité pour les bananiers sont : $\delta 124, \delta 125, \delta 15, \delta 85, \delta 91, \delta 102, \delta 104$, $\delta 108, \delta 110, \delta 117, \delta 147, \delta 56, \delta 66, \delta 77, \delta 51, \delta 52$. Un effet souche a été observé sur le phytotoxicité des filtrats de culture de M. fijiensis. En effet, la toxicité des filtrats de culture a varié selon la souche de $M$. fijiensis avec des différences significatives $(P<0,001)$. Le filtrat de culture de la souche $\delta 15$ a induit des nécroses foliaires avec l'indice le plus élevé $(3,02)$ tandis que les filtrats des 
souches $\delta 23, \delta 35, \delta 126, \delta 127, \delta 63, \delta 115, \delta 106, \delta 100$,

(Figure 1).

$\delta 123$ et $\delta 98$ n'ont pas été toxiques pour les bananiers

Tableau 3 : Composition chimique des filtrats de culture des souches de M. fijiensis

\begin{tabular}{|c|c|c|c|c|c|c|c|}
\hline \multirow{2}{*}{$\begin{array}{l}\text { Souches } \\
\text { fongiques }\end{array}$} & \multicolumn{7}{|c|}{ Composés chimiques recherchés } \\
\hline & Saponines & $\begin{array}{c}\text { Stérols } \\
\text { terpènes }\end{array}$ & Quinones & Polyphénols & Flavonoïdes & Tanins & Alcaloïdes \\
\hline $\mathrm{T}_{0}$ & - & - & - & - & - & - & - \\
\hline$\delta 51$ & ++ & +t & - & - & - & - & ++ \\
\hline$\delta 52$ & + & ++ & - & - & - & - & ++ \\
\hline$\delta 77$ & ++ & +t & - & - & - & - & + \\
\hline$\delta 23$ & - & + & - & - & - & - & + \\
\hline$\delta 35$ & ++ & + & - & - & - & - & ++ \\
\hline$\delta 126$ & t++ & + & - & - & - & - & ++ \\
\hline$\delta 127$ & +++ & + & - & - & - & - & t++ \\
\hline$\delta 63$ & t++ & t++ & - & - & - & - & t++ \\
\hline$\delta 66$ & ++ & ++ & - & - & - & - & t+ \\
\hline$\delta 56$ & - & - & - & - & - & - & + \\
\hline$\delta 117$ & ++ & + & - & - & - & - & + \\
\hline$\delta 147$ & ++ & + & - & - & - & - & + \\
\hline$\delta 115$ & + & - & - & - & - & - & +++ \\
\hline$\delta 106$ & + & ++ & - & - & - & - & ++ \\
\hline$\delta 108$ & + & + & - & - & - & - & + \\
\hline$\delta 100$ & + & + & - & - & - & - & ++ \\
\hline$\delta 102$ & ++ & +++ & - & - & - & - & +++ \\
\hline$\delta 104$ & ++ & ++ & - & - & - & - & + \\
\hline$\delta 85$ & + & + & - & - & - & - & ++ \\
\hline$\delta 15$ & ++ & + & - & - & - & - & + \\
\hline$\delta 124$ & + & + & - & - & - & - & + \\
\hline$\delta 125$ & +++ & + & - & - & - & - & ++ \\
\hline$\delta 123$ & - & + & - & - & - & - & + \\
\hline$\delta 98$ & +++ & + & - & - & - & - & + \\
\hline$\delta 91$ & +++ & + & - & - & - & - & + \\
\hline$\delta 110$ & + & + & - & - & - & - & ++ \\
\hline
\end{tabular}

$\mathrm{T}_{0}=$ milieu non inoculé par $M$. fijiensis ; = Absence de réaction ; + = réaction faible $; \boldsymbol{+ +}=$ réaction moyennement intense $; \boldsymbol{+ + +}=$ réaction très intense 


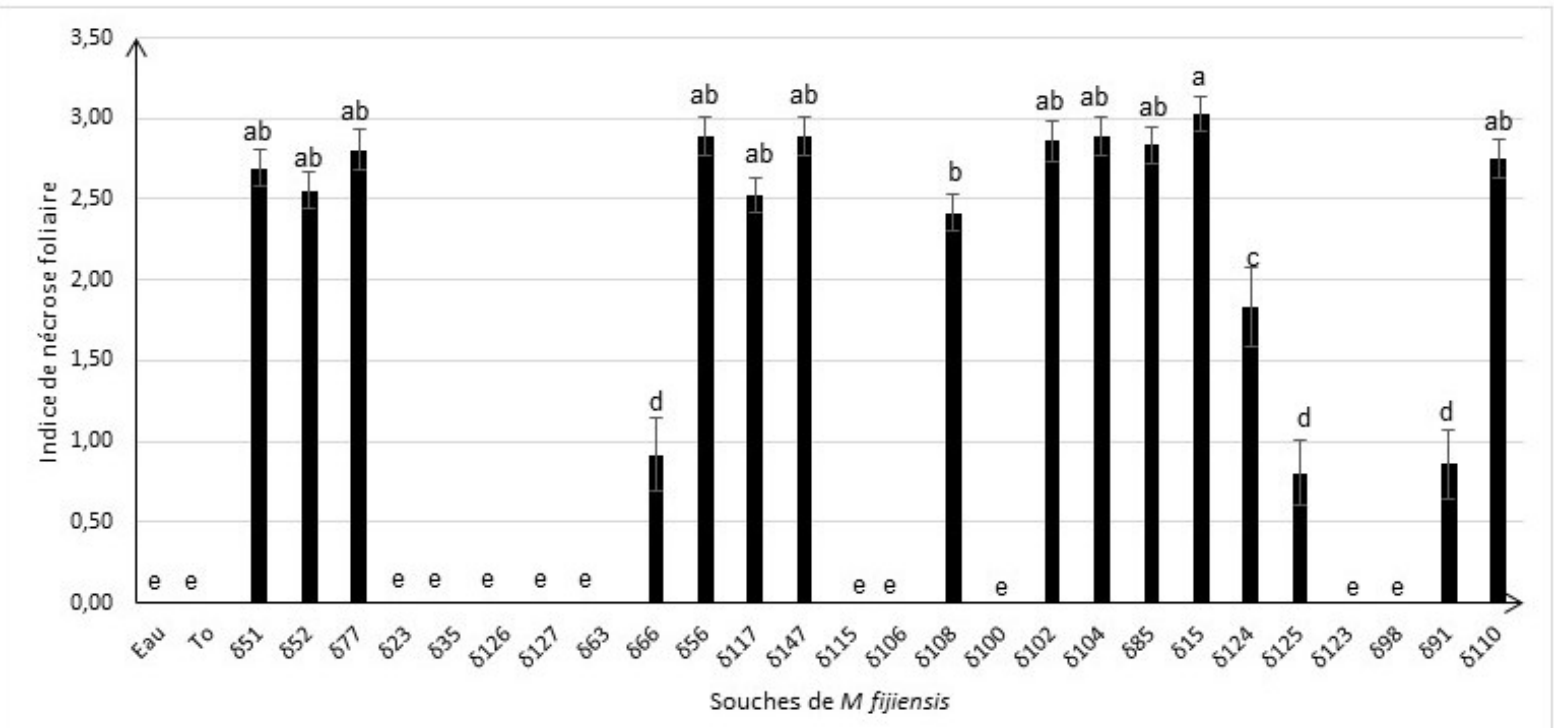

Figure 1 : Effets des souches de M. fijiensis sur l'indice de nécroses foliaires des bananiers 48 heures après infiltration des filtrats de culture

Toutes les variétés de bananiers y compris Calcutta 4 (très résistant à l'infection de $M$. fijiensis), se sont révélées sensibles à l'induction des nécroses après infiltration des filtrats de culture des souches fongiques (Figure 2). Un effet variétal a été observé dans la réaction des bananiers contre la toxicité des filtrats de culture de $M$ fijiensis avec des différences hautement significatives $(P<0,0001)$. La variété FHIA 23 a présenté une sensibilité plus faible à la toxicité des filtrats de culture, avec l'indice de nécrose foliaire le plus faible $(1,03)$. Cependant, les génotypes Grande Naine et Calcutta 4 se sont montrés plus sensibles à la toxicité des filtrats de culture $M$ fijiensis, avec des indices de nécrose foliaires respectifs de 1,31 et 1,67 (Figure 2). L'effet de l'interaction entre les souches de M fijiensis et les variétés de bananier sur l'indice de nécroses foliaires, est caractérisé par les valeurs les plus faibles $(2,08)$ pour la combinaison souche $\delta 108 / F I H A 23$ et les plus élevées $(3,41)$ pour la combinaison souche $\delta 147 /$ Calcutta 4 (Figure 3). Dans l'ensemble, les nécroses foliaires formées dans les combinaisons incluant la variété Calcutta 4 ont présenté des indices plus élevés (2,41 3,41 ), contrairement à celles provenant des combinaisons intégrant la variété FIHA $23(2,08-2,75)$. Pour les combinaisons comportant la variété Grande Naine, les valeurs d'indices de nécroses foliaires ont été intermédiaires aux précédentes et comprises entre 2,25 et 3,16 (Figure 3). Les différences observées ont été significatives $(P<0,001)$ au seuil de $5 \%$. 


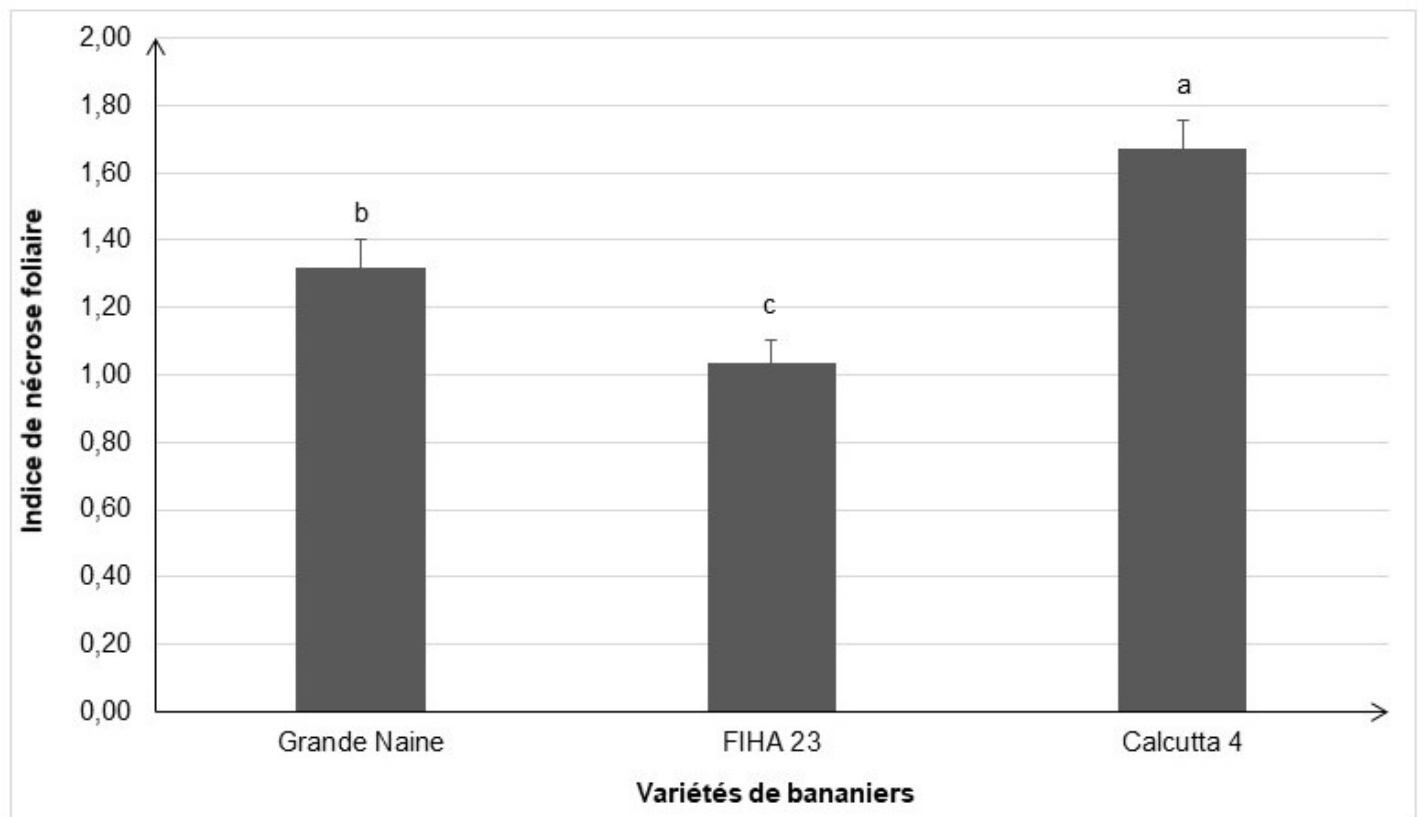

Figure 2 :Effets des variétés de bananier sur l'indice de nécroses foliaires 48 heures après infiltration des filtrats de culture de M. fijiensis

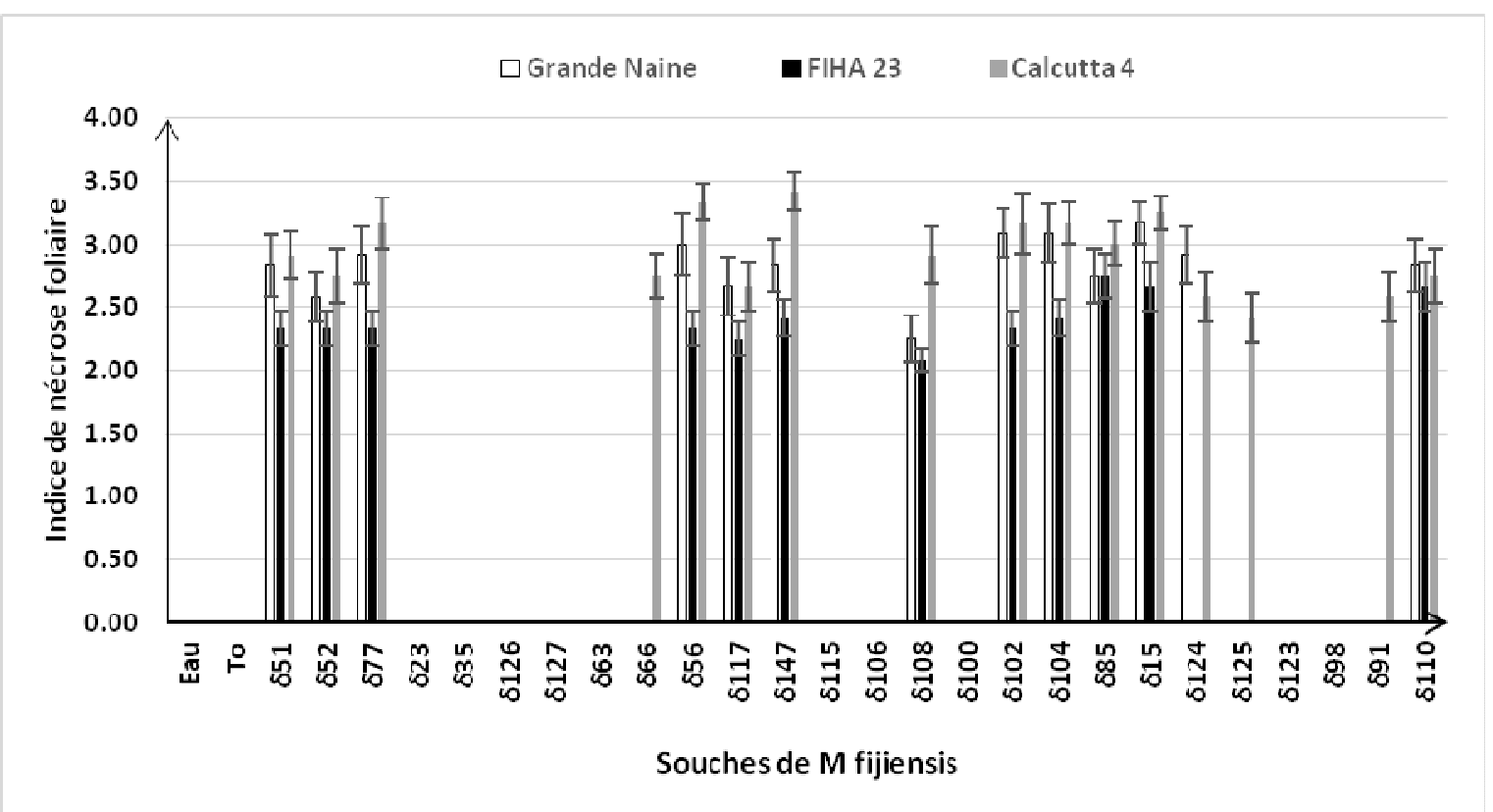

Figure 3 : ndice de nécroses foliaires des génotypes de bananières 48 heures après infiltration des filtrats de culture des différentes souches de M. fijiensis

Distribution des métabolites secondaires dans les filtrats de culture $M$ fijiensis et réaction des variétés de bananiers à la phytotoxicité : Dans toutes les principales régions productrices de la banane en Côte d'Ivoire, il a été retrouvé des souches de $M$ fijiensis dont les filtrats de culture ont pu induire des nécroses sur les feuilles de bananiers (Tableau 4). L'ensemble des 16 souches dont les filtrats de culture ont montré une phytotoxicité vis-à-vis des variétés de bananiers testées le sont en particulier pour la variété Calcutta 4. 
Cependant, parmi les 16 filtrats phytotoxiques, les variétés de bananiers Grande Naine et FHIA 23 ont enregistré respectivement $13(50,00 \%)$ et $12(46,15 \%)$ souches de $M$ fijiensis dont les filtrats de culture provoquent la formation de nécroses foliaires (Tableau 4). La présence d'au moins un des trois groupes de composés secondaires (saponines, stérols et terpènes, alcaloïdes) mis en évidence dans les filtrats de culture, a été observée chez les souches de $M$ fijiensis dont les filtrats ont été phytotoxiques pour les bananiers (Tableau 4). Toutefois, aucune nécrose n'a été observée sur les feuilles des bananiers avec certaines souches de $M$ fijiensis $(\delta 98, \delta 100, \delta 106, \delta 35, \delta 126, \delta 127$ et $\delta 63)$ dont les filtrats de cultures contenaient tous les trois métabolites secondaires mis en évidence (Tableau 4). Les alcaloïdes ont été retrouvés dans tous les filtrats de culture phytotoxiques pour les bananiers, en particulier avec la souche $\delta 56$ qui n'a produit ni les saponines, ni les stérols et terpènes. Également, la présence des alcaloïdes a été observée dans tous les filtrats de cultures qui n'ont pas induit de nécroses sur les feuilles des bananiers (Tableau 4). Cependant, parmi ces filtrats de culture non-phytotoxiques, certaines souches de $M$ fijiensis n'ont pas pu produire les composés du groupe des saponines et/ou des stérols et terpènes $(\delta 115, \delta 123$, ס23).

Tableau 4 : Composition des filtrats de culture des souches de $M$. fijiensis issues des principales régions de production de bananes en Côte d'Ivoire et réaction de différentes variétés de bananiers à l'effet des filtrats de culture

\begin{tabular}{|c|c|c|c|c|c|c|c|}
\hline \multirow{2}{*}{$\begin{array}{l}\text { Souches } \\
\text { de } M \text {. } \\
\text { fijiensis }\end{array}$} & \multirow[t]{2}{*}{$\begin{array}{l}\text { Régions de la } \\
\text { Côte d'Ivoire }\end{array}$} & \multicolumn{3}{|c|}{$\begin{array}{c}\text { Composés secondaires des filtrats de } \\
\text { cultures }\end{array}$} & \multicolumn{3}{|c|}{ Variétés de bananiers } \\
\hline & & Saponines & $\begin{array}{l}\text { Stérols et } \\
\text { terpènes }\end{array}$ & Alcaloïdes & $\begin{array}{l}\text { Grande } \\
\text { Naine }\end{array}$ & FHIA 23 & Calcutta 4 \\
\hline$\delta 124$ & Sud Est & + & + & + & + & - & + \\
\hline$\delta 125$ & Sud Est & + & + & + & - & - & + \\
\hline$\delta 15$ & Sud & + & + & + & + & + & + \\
\hline$\delta 85$ & Sud & + & + & + & + & + & + \\
\hline$\delta 91$ & Sud & + & + & + & - & - & + \\
\hline$\delta 98$ & Sud & + & + & + & - & - & - \\
\hline$\delta 100$ & Sud & + & + & + & - & - & - \\
\hline$\delta 102$ & Sud & + & + & + & + & + & + \\
\hline$\delta 104$ & Sud & + & + & + & + & + & + \\
\hline$\delta 106$ & Sud & + & + & + & - & - & - \\
\hline$\delta 108$ & Sud & + & + & + & + & + & + \\
\hline$\delta 110$ & Est & + & + & + & + & + & + \\
\hline$\delta 115$ & Est & + & - & + & - & - & - \\
\hline$\delta 35$ & Sud Ouest & + & + & + & - & - & - \\
\hline$\delta 123$ & Sud Ouest & - & + & + & - & - & - \\
\hline$\delta 117$ & Sud Ouest & + & + & + & + & + & + \\
\hline$\delta 147$ & Sud Ouest & + & + & + & + & + & + \\
\hline$\delta 23$ & Centre Ouest & - & + & + & - & - & - \\
\hline$\delta 126$ & Centre Ouest & + & + & + & - & - & - \\
\hline$\delta 127$ & Centre Ouest & + & + & + & - & - & - \\
\hline$\delta 56$ & Centre Ouest & - & - & + & + & + & + \\
\hline$\delta 63$ & Ouest & + & + & + & - & - & - \\
\hline$\delta 66$ & Ouest & + & + & + & - & - & + \\
\hline$\delta 77$ & Ouest & + & + & + & + & + & + \\
\hline$\delta 51$ & Ouest & + & + & + & + & + & + \\
\hline$\delta 52$ & Ouest & + & + & + & + & + & + \\
\hline
\end{tabular}

$+=$ Présence du composé chimique dans le filtrat de culture ou induction de nécrose sur la feuille du bananier après infiltration du filtrat de culture

- = Absence du composé chimique dans le filtrat de culture ou absence de nécrose sur la feuille du bananier après infiltration du filtrat de culture 


\section{DISCUSSION}

Croissance mycélienne, $\mathrm{pH}$ et métabolites secondaires produits dans les filtrats de culture des souches de M. fijiensis issues de la Côte d'Ivoire : La variabilité des propriétés physico-chimiques observée entre les différentes souches utilisées dans cette étude, montre que celles-ci ne réagissent pas toutes de la même façon dans le milieu de culture MD1 modifié. II apparaît également que les filtrats ayant enregistré une bonne croissance mycélienne ne sont pas forcément ceux qui ont des rendements élevés en extrait organique brut. Ces résultats suggèrent que chez $M$. fijiensis, il n'existe aucune relation entre la quantité de mycélium produite par une souche donnée dans une culture et la synthèse efficace des métabolites secondaires, (Upadhyay et al. 1991 ; Puch-Ceh et al. 2005). En effet, la souche $\delta 115$ qui a produit la plus grande masse mycélienne n'est pas la plus productrice d'extrait organique. Inversement la souche $\delta 147$ qui a produit le plus d'extrait organique brut est celle qui présente la plus faible quantité de mycélium. Aussi, la production de métabolites semble être dépendante de façon intrinsèque de la souche et non de sa croissance. Le faible $\mathrm{pH}$ des filtrats de culture qui favorise la croissance mycélienne, indique que certains composés (toxiques ou non) excrétés par Mycosphaerella fijiensis seraient de nature acide, ou des molécules facilement acidifiables. Des résultats similaires ont été rapportés par Lépengué (2008) sur Phoma sabdariffae Sacc., agent pathogène de la roselle (Hibiscus sabdariffa L. var. sabdariffa). En effet, les agents pathogènes excrètent concomitamment aux substances toxiques, des molécules diverses dont certaines joueraient un rôle de stabilisateurs des substances toxiques (Harelimana, 1997). L'acidification des milieux de culture pourrait alors constituer un facteur impliqué dans les mécanismes de résistance faisant intervenir des formes résistantes de la cible moléculaire des toxines synthétisées (Boucher et al., 2001). II convient de noter que l'aptitude d'un agent pathogène à produire une toxine est parfois associée à la présence, chez ce pathogène, de fonctions qui lui permettent d'échapper aux effets délétères de sa propre toxine. La toxicité de la molécule n'est rétablie qu'après libération de celle-ci dans la plante suite à une oxydation spontanée. La variabilité entre les métabolites secondaires produits pourrait refléter des différences de cinétique de croissance liée au génotype de chaque isolat. Également, cette production variable de métabolites pourrait être en rapport avec la virulence et l'agressivité des souches. Par ailleurs, la présence des alcaloïdes a été mise en évidence dans les filtrats de culture de souches de Cladosporium musae et de
Deigthoniella torulosa, des champignons responsables d'attaques foliaires chez le bananier (Koné, 2008). Etamé (2003) dans ces travaux, a caractérisé les métabolites des extraits toxiques bruts (ETB) des filtrats de culture de souches de M. fijiensis issues du Cameroun, Cet auteur a mis en évidence en plus des molécules déjà identifiées telle la juglone, d'autres composés dont le profil HPLC ne correspondait à aucun des métabolites déjà rapportés chez ce pathogène. Parmi ces molécules non encore signalées comme métabolites secondaires de $M$. fijiensis se trouvent les esters d'acide gras auxquels s'apparentent les saponosides mis en évidence dans la présente étude avec les filtrats de culture des souches ivoiriennes.

Sensibilité des bananiers aux filtrats de culture des souches de $M$. fijiensis : La formation de nécroses sur le limbe foliaire, suggère l'existence d'une ou plusieurs substances toxiques dans les filtrats de culture des souches de M. fijiensis. Les travaux de différents auteurs ont permis également de mettre en évidence des substances toxiques dans les filtrats de culture de quelques souches de $M$. fijiensis essentiellement originaires de l'Asie, de l'Amérique (Stierle et al., 1991, Strobel et al., 1993, Harelimana et al., 1997 ; Puch-Ceh et al, 2005). Le test d'induction de nécroses a révélé des indices nécrotiques variables entre les différentes variétés de bananiers. Ces résultats indiquent que les différences de toxicité seraient liées tout au moins, à des différences qualitatives et/ou à un degré moindre, à une variabilité quantitative dans la composition chimique des filtrats de culture des souches de $M$ fijiensis. En outre, l'éventualité d'une action synergique des différents métabolites dans les filtrats de culture s'illustre parfaitement avec ce biotest. Toutefois, la présence des trois groupes de composés (saponines, stéroles et terpènes et les alcaloïdes) n'a pas toujours suffi pour induire les nécroses après infiltration des filtrats dans les limbes foliaires. Les filtrats non toxiques pourraient appartenir à des souches de $M$. fijiensis non pathogènes qui d'après Harelimana (1997), ont des extraits bruts très peu toxiques comparés à ceux des souches pathogènes. La formation des lésions nécrotiques suite à l'action des filtrats de culture infiltrés dans les feuilles, indique une interaction entre les métabolites toxiques produits par les souches de M. fijiensis et les systèmes antioxydants. Une telle interaction entre toxines et antixoydants permet de supposer la production des formes actives d'oxygène (Amari et al., 2011). L'établissement de cette preuve expliquerait l'apparition des nécroses suite à l'infiltration des filtrats de culture, d'autant plus que les formes actives 
d'oxygène sont susceptibles de produire des nécroses (Busogoro et al., 2002). L'hybride FHIA 23, partiellement résistant à $M$. fijiensis, a été moins sensible à la toxicité des filtrats de culture contrairement aux variétés Grande Naine et Calcutta 4 respectivement sensible et très résistant à $M$. fijiensis. La résistance totale chez Calcutta 4 serait sans relation avec les toxines produites par les souches de M. fijiensis. Des résultats similaires ont été obtenus par Harelimana et al. (1997) sur le clone Yangambi Km5, hautement résistant à la maladie des raies noires avec des extraits bruts de souches de $M$. fijiensis originaires du Honduras. En effet, contrairement à la résistance partielle ou horizontale dite polygénique et stable dont les mécanismes s'exprimeraient au cours de la phase nécrotrophe (cas de l'hybride FHIA 23), la résistance totale ou verticale (cas de Calcutta 4) quant à elle, est habituellement oligogénique et instable surtout

\section{CONCLUSION}

Cette étude a permis de mettre en évidence la sécrétion de métabolites toxiques par les différentes souches de $M$. fijiensis en provenance des principales régions productrices de bananes en Côte d'lvoire. Ces métabolites secondaires appartiennent principalement aux groupes des saponines, des stérols et terpènes et des alcaloïdes. Au cours de cette production des toxines, la croissance du mycélium est négativement corrélée au $\mathrm{pH}$ du filtrat de culture du champignon. Cependant, seulement certains filtrats de cultures du champignon ont pu induire des nécroses après leur infiltration dans les feuilles des différentes variétés de bananiers. La

\section{REMERCIEMENTS}

Les auteurs remercient les responsables de la mycothèque du Laboratoire de Physiologie Végétale de l'Université Félix Houphouêt-Boigny d'Abidjan et les

\section{REFERENCES BIBLIOGRAPHIQUES}

Amari L.D.G.E., Koné, Dick E., Traoré S., Kobenan K. \& Abo A.P., 2008. Etude comparée de paramètres photosynthétiques chez différentes variétés de bananiers après infiltration de la juglone, un métabolite toxique de Mycosphaerella fijiensis, agent causal de la cercosporiose noire. Journal of Applied Biosciences, Vol. 10 (2): 523 - 531.

Amari L.D.G.E., DICK E., Koné D., Abo K. \& Kobenan K., 2011. Response to juglone toxic effect in various genotypes of banana (Musa AA, AAA, AAB, AAAA, AAAB). Agronomie Africaine 23 (3) : $247-258$. dans une zone où l'agent pathogène présente une variabilité génétique importante. La résistance/sensibilité aux toxines est donc un mécanisme qui ne se révèle qu'après la phase biotrophe de l'infection et concernerait la phase nécrotrophe pendant laquelle s'exprime la résistance partielle (Harelimana, 1997 ; Amari et al., 2014). Un probable contournement de la résistance de Calcutta 4 est à envisager à l'instar de Yamgambi Km5, une autre variété de bananier pourtant réputée très résistante à M. fijiensis mais qui depuis 1999 est attaquée par la MRN. L'étude des métabolites toxiques induisant les symptômes dus à $M$. fijiensis responsable de la cercosporiose noire, confirme bien la présence de souches pathogènes du champignon dans toutes les principales zones productrices de bananes en Côte d'Ivoire y compris la région des Montagnes où elle n'était pas signalée auparavant (Traoré, 2008 ; Camara, 2011).

résistance du clone de bananier, Calcutta 4 à $M$. fijiensis est indépendante de la résistance aux toxines produites par ce pathogène. L'hybride FHIA 23, partiellement résistant à $M$. fijiensis a montré un niveau de résistance plus élevé à la toxicité des filtrats de culture du champignon. La production de métabolites secondaires par $M$. fijiensis constitue un facteur déterminant du contournement de la résistance variétale chez le bananier par ce champignon. La présence de souche pathogène du champignon est à signaler dans toutes les principales zones productrices de bananes en Côte d'Ivoire.

techniciens pour la mise à disposition des souches de Mycosphaerella fijiensis.

Amari L.D.G.E., Chérif M., KOUAKOU T.H., Camara B. And Koné D. (2014). Salicylic acid and acibenzolar-s-methyl induced Resistance against toxic effect of juglone, a toxin of Mycosphaerella fijiensis causal agent of banana black leaf streak disease. Journal of Advances in Agriculture 3 (3) 204-217.

Boucher C., Genin S. \& Arlat M., 2001. Concepts actuels sur la pathogénie chez les bactéries phytopathogènes. CR Acad. Sci. Paris, Sciences de la vie/Life sciences, 324 : 915-922. Busogoro J.P., Panis B., Messiaen J., van Custem P., Swennen R. \& Lepoivre P., 2002. Evènements 
oxydatifs induits par les métabolites de Mycosphaerella fijiensis chez la cercosporiose noire du bananier (Musa spp.) et analyse de la faisabilité d'une sélection précoce de la résistance à cette maladie. Promusa 9 : XXIV.

Camara B., 2011. Caractérisation des parasites fongiques foliaires et telluriques en Côte d'Ivoire chez les bananiers (Musa sp.) et recherche de méthodes de lutte. Thèse de Doctorat Unique. Laboratoire de Physiologie Végétale, UFR biosciences, Université de Cocody-Abidjan. 219 p.

Canãs-Gutiérrez, P.G., Angarita-Velásquez, M.J., Restrepo-Flórez, J.M., Rodríguez, P., Moreno, C.X \& Arango, R., 2009. Analysis of the CYP51 gene and encoded protein in propiconazoleresistant isolates of Mycosphaerella fijiensis. Pest Manag Sci ; 65 :892-899.

Carlier J., Zapater M.F, Lapeyere F., Jones D.R. \& Mourichon X., 2000. Septoria leaf Spot of Banana : A newly Discovered Disease Caused by Mycosphaella eumusae (anamorph Septoria eumusae). Phytopathology, 90: 884-890.

El Hadrami A, Koné D, \& Lepoivre P., 2005. Effect of juglone on active oxygen species and antioxidant enzymes in susceptible and partially resistant banana cultivars to Black Leaf Streak Disease. European Journal of Plant Pathology 113: $241-254$

Essis, B., Kobenan, K., Traoré, S., Koné, D. \& Yatty, J., 2010. Sensibilité au laboratoire de Mycosphaerella fijiensis responsable de la cercosporiose noire des bananiers vis-à-vis de fongicides couramment utilisés dans les bananeraies ivoiriennes. Journal of Animal \& Plant Sciences. Vol. 7, Issue 2: 822-833.

Etamé J.J., 2003. Caractérisation des toxines de Mycosphaerella fijiensis, l'agent de la cercosporiose noire du bananier pour leur utilisation comme agent de sélection précoce de variétés de bananier résistantes. Ph. D, Faculté Universitaire des Sciences agronomiques de Gembloux (FUSAGx), $171 \mathrm{p}$.

Ganry J., 2010. Black Leaf Streak Disease in Martinique. Fruits, 65: 325.

Harelimana G., Lepoivre P., Jijakli \& Mourichon X., 1997. Use of Mycosphaerella fijiensis toxins for the selection of banana cultivars resistant to Black Leaf Streak. Euphytica 96: 125-128.

Koné D., 1998. Contribution à l'étude des cercosporioses et des cladosporioses des bananiers en Côte d'lvoire. Thèse de Doctorat $3^{\text {ème }}$ cycle. Laboratoire de Physiologie Végétale, UFR Biosciences, Université de Cocody-Abidjan, 245 $p$.

Koné D. (2008). Contribution à l'étude des champignons epiphylles des bananiers en Côte d'Ivoire: Caréctéristion morphologique et pathologique de Mycosphaerella fijiensis var. difformis Morelet, Cladosporium musae Mason, Deightoniella turulosa (Syd.) Ellis et Cordana musae Zim. ; sensibilité à quelques fongicides. Thèse de Doctorat d'Etat. Laboratoire de Physiologie Végétale, UFR Biosciences, Université de Cocody-Abidjan $244 \mathrm{p}$.

Lépengué A.N., 2008. Contribution à l'étude du filtrat de culture de Phoma sabdariffae Sacc., agent pathogène de la roselle (Hibiscus sabdariffa $\mathrm{L}$. var. sabdariffa), au Gabon. Thèse de Doctorat Unique. Laboratoire de Physiologie Végétale, UFR Biosciences, Université de CocodyAbidjan. $267 \mathrm{p}$.

Puch-Ceh M., Gracia-Sosa K. \& Peña-Rodriguez L.M., 2005. Optimisation des conditions de culture de Mycosphaerella fijiensis Morelet. InfoMusa, 14 (1) : 21-23.

Soler A, Alphonsine PM, Corbion C, MarieLuce S. and Quénéhervé P., 2012. Les défenses naturelles des plantes contre les bioagresseurs: Un nouvel atout dans la mise au point de systèmes de cultures plus écologiques. Cahiers du PRAM.;11(12):31-34.

Stierle A.A., Upadhayay, Hershenhorn J., Strobel G.A. \& Molina G., 1991. The phytotoxins of Mycosphaerella fijiensis, the causative agent of black Sigatoka disease of bananas and plantains. Experientia 47: 853-859.

Strobel G.A., Stierle A.A., Upadhyay R., Hershenhorn J. \& Molina G., 1993. The phytotoxines of Mycosphaerella fijiensis, the causative agent of Black Sigatoka disease and their use in screening for disease resistance. In: Biotechnology applications for banana and plantain improvement. Fullerton R.A. and Stover R.H. (Eds). Montpellier France. Pp 93-103.

Tarik C., Farah H. \& Fawzia A.B., 2011. Phytochemical study of roots and leaves of the plant Echlumpycnanthum pommel. Der Pharmacia Lettre, 3 (2) 1-4.

Traoré S., Kobenan K., Kendia E., Koné D. \& Traoré D., 2008. Relation entre densité stomatique et réaction à la maladie des raies noires chez 
différents génotypes de bananiers et de bananiers plantain. Agronomie Africaine. Vol 20

(1) $37-47$.

Upadhyay R., Strobel G., Coval S. \& Clardy J., 1991. Fijiensin, the first phytotoxin from Mycosphaerella fijiensis, the causative agent of black sigatoka disease. Experientia, 47: 982984.

Vishnevetsky, J., White, T.L., Palmateer, A.J., Flaishman, M., Cohen, Y., Elad, Y., Velcheva, M., Hanania, U., Sahar, N., Dgani, O. \& Perl, A., 2011. Improved tolerance toward fungal diseases in transgenic Cavendish banana (Musa spp. AAA group) cv. Grand Nain. Transgenic Res., 20:6172. 